\title{
Probe timing optimization for time-reversal underwater communications
}

\author{
António Silva and Sérgio M. Jesus \\ João Gomes \\ Institute for Systems and Robotics, Universidade do Algarve Institute for Systems and Robotics, Instituto Superior Técnico \\ Campus de Gambelas, PT-8005-139 Faro, Portugal \\ Email: \{asilva,sjesus\}@ualg.pt \\ Av. Rovisco Pais, PT-1049-001 Lisboa, Portugal \\ Email: jpg@isr.ist.utl.pt
}

\begin{abstract}
Passive Time Reversal (pTR) is one of the variants of time reversal applicable to digital underwater communications. In passive time reversal a probe-signal is transmitted ahead of the data-signal in order to estimate the channel impulse response for later use as a replica signal in a time reversal mirror fashion. In practice the received probe-signal must be captured in a time-window and, after correlation with the transmitted probesignal, give a noisy estimation of the channel impulse response. Therefore, the output Signal to Noise Ratio (SNR), the InterSymbolic Interference (ISI) and the detection rate of passive time reversal will strongly depend on the starting time and duration of such time-window. Typically the beginning and the duration of that time-window should depend on the travel time and the dispersion of the acoustic channel. In this paper, the maximization of the pTR output SNR relative to the probe time-window is derived in closed form. It will be shown that the probe timing that gives the lower detection error rate can be predicted using closed form metrics for the pTR output SNR and ISI. Theoretical results are found to be in full agreement with simulations and with results obtained on experimental data taken during the INTIFANTE'00 sea trial.
\end{abstract}

\section{INTRODUCTION}

In the past few years coherent modulation techniques for fast and reliable shallow water acoustic communication have triggered a number of theoretic developments, simulations and field experiments. To that end multichannel adaptive equalization methods [1], although quite computationally demanding, currently provide the most popular framework. Recently, active and passive Time Reversal (a-pTR) [2], [3] appeared as a viable alternative for simple and robust underwater coherent communications [4]-[6]. Active Time Reversal (aTR) takes advantage of the acoustic channel mode orthogonality and reciprocity properties and matches the ocean response with itself. Like aTR, passive Time Reversal (pTR) relies on mode orthogonality but instead of the reciprocity property, uses an estimate of the underwater channel Impulse Responses (IRs) to perform a virtual ocean response match inside the computer. Despite its simplicity, a-pTR applied to high frequency underwater communications presents a lower performance than multichannel equalization [7]-[9]. That is due to the Time Reversal Mirror (TRM) requirement for a long and dense array [10], without which residual Inter-Symbol Interference (ISI) is always present due to poor sampling of the highorder modes and subsequent orthogonality property violation.
One of the most critical aspects of the a-pTR methods is the channel IR estimation, which is typically obtained by simply correlating the received channel distorted probe-signal with the transmitted one, resulting in a noisy version of the channel IR.

Figure 1 shows a block diagram of the pTR application adopted in the sequel, where the received probe-signal $f_{i}^{\prime}(t)$ is the channel IR estimate that is simply obtained as the channel noise contaminated response to a dirac impulse (upper path in the block diagram). For later use the estimated IR must be approximated by a FIR filter, which means that it must be captured in a finite time-window (see Figure 1). The time windowed estimated IR, $g_{i, t 0, \tau}(t)$, is them used as a matched filter with the received data signal $v_{i}(t)$ (lower path in the block diagram). The start time and the duration of such timewindow should depend on the time dispersion of the acoustic channel. Heuristic reasoning would suggest that if a short timewindow fails to include all significant multipath it will result in an imperfect retrofocusing, while a too long time-window will reduce the efficiency of the communication system and introduce additional noise in the pTR operation [5], [7], [11].

For a well designed pTR Vertical Line Array ${ }^{1}$ that is able to reduce the residual ISI to an acceptable level, the time-window optimization can be transformed into a problem of pTR output SNR maximization, that can be solved after establishing signal and noise power time-window dependence. The a-pTR output SNR have been addressed by several authors [9], [12], including heuristic characterizations of time-window dependence [5], [7], [11] though optimization was not attempted.

When the VLA configuration is not able to reduce ISI to an acceptable level the global optimal time-window become dependent of both: ISI and pTR output SNR metrics. The time-widow length controls the amount of pTR IR spread that is considered for the ISI and pTR output SNR computation. It turns out that in order to optimize the communication system performance the ISI should be minimized while the pTR output SNR should be maximized with respect to the time-window length. It will be shown that the optimal pTR output SNR occurs when the time-windowed pTR overall IR has maximum power, while the optimal time-window for ISI

\footnotetext{
${ }^{1}$ i.e., that there is a sufficiently large number of hydrophones, the vertical array is spanning the whole water column and the propagation environment is time-invariant.
} 
tends to occur when the full length of the pTR overall IR is considered. Moreover, it will be shown that the time-window that gives an optimum Mean Square Error (MSE), between the transmitted and estimated symbols sequence, can be predicted by the pTR output SNR when in presence of a low input SNR, and by the ISI when in presence of a high input SNR.

In Section II signal and noise terms of the pTR communication system are identified and their means and powers are derived (a full derivation can be found in [13]). In Section III a closed form expression for the pTR output SNR as a function of the time-window is obtained and strategies for its optimization are proposed. In particular, it is found that the optimal time-window does not depend on the input noise level but only on the multipath structure of the underwater acoustic channel. Section IV presents the results obtained in simulation using realistic underwater acoustic propagation models. In Section V the proposed optimization method will be applied to real data acquired during the INTIFANTE'00 sea trial. Conclusions and future work are presented in Section VI

\section{IDEAL PTR COMMUNICATION SYSTEM}

The objective of this Section is to set up the theoretical background for analysing the implications of probe-signal windowing operation in pTR performance when applied to digital communications in the presence of acoustic noise. An 'ideal' pTR where the TRM basic associated assumptions are fulfilled will be considered. In such conditions the ISI at the pTR communication system output is negligible and the system performance is only constrained by the pTR output SNR.

\section{A. Digital communications with passive Time Reversal}

Figure 1 shows the baseband equivalent of the sourcechannel-receiver representation of the pTR processor for one hydrophone. In a first step (upper path in Figure 1) a duly time windowed and phase conjugated channel IR estimate is computed. In a second step (lower path in Figure 1) the deconvolution of the transmitted data sequence $a_{n}$ distorted by the underwater channel is accomplished using the estimated channel IR computed in the first step. In that figure, the transmitting and receiving filter, $p(t)$, is a fourth-root raised cosine pulse. In the sequel

$$
p_{m}(t)=\underbrace{p(t) * \ldots * p(t)}_{\mathrm{m} \text { times }},
$$

represents the m-times self-convolution of $p(t)$ such that $p_{4}(t)$ is the raised-cosine pulse shape function. In the IR estimation step, $p_{2}(t)$ is used as a narrowband filter resulting in a squareroot raised cosine shape. In the second step $p(t)$ is used as the transmitting pulse shape for the data sequence that, in conjunction with $p(t)$ in the receiver side, results in a received data sequence square-root raised cosine pulse shaped, distorted with the baseband equivalent channel IR $h_{i}(t)$. With such configuration, in presence of a non-distortive channel (that is $\left.h_{i}(t)=h_{i}^{\prime}(t)=\delta(t)\right)$ and with a sufficiently large timewindow, one can guarantee a raised cosine pulse shape for the data sequence in the pTR output signal $z(t)$.

Let us assume that the transmitted signal is Pulse Amplitude Modulated (PAM) written as

$$
s(t)=a(t) * p(t),
$$

with

$$
a(t)=\sum_{n=-\infty}^{+\infty} a_{n} \delta\left(t-n T_{b}\right),
$$

where $a_{n}$ is a zero mean symbol sequence assumed to be white with power $\sigma_{a}^{2}$, and $T_{b}$ is the symbol duration.

Assuming the acoustic channel as a time-invariant linear system with impulse response $h_{i}(t)$, the received data-signal at hydrophone $i$ is given by

$$
v_{i}(t)=h_{i}(t) * a(t) * p_{2}(t)+w_{i}(t) * p(t),
$$

where $w_{i}(t)$ is an additive zero mean white noise with power $\sigma_{w}^{2}$, assumed to be uncorrelated with the signal and from sensor to sensor. When the probe-signal is a dirac impulse the received probe (upper path in Figure 1) is written as

$$
f_{i}^{\prime}(t)=h_{i}^{\prime}(t)+u_{i}(t)
$$

where $u_{i}(t)$ is the channel additive noise sequence with the same properties as $w_{i}(t)$ and independent from it, $h_{i}^{\prime}(t)$ is the same channel impulse response as $h_{i}(t)$ (no environment/geometry mismatch case) and the ' denotes that there is an unspecified time delay between the two impulse responses (IRs).

The time-window operator multiplies the input signal with a unit-gate function of length $\tau$ and starting point $t_{0}$, resulting $f_{i, t_{0}, \tau}^{\prime}(t)$ that after being filtered by $p_{2}(t)$ gives the narrowband time-limited IR estimate $g_{i, t_{0}, \tau}(t)$.

Finally, the narrowband time limited IR estimate is phase conjugated or, equivalently in the time domain, time-reversed and conjugated. The pTR output for channel $i$ is therefore

$$
z_{i}(t)=g_{i, t_{0}, \tau}^{*}(-t) * v_{i}(t)
$$

where $v_{i}(t)$ is given by (4). Summing (6) over the hydrophone index $i$, the pTR output signal can be written as

$$
z(t)=y(t)+H U(t)+H W(t)+U W(t),
$$

where $y(t)$ contains the desired data-signal contaminated with residual ISI and the other three terms are noise disturbances, defined as

$$
\begin{aligned}
y(t) & =\sum_{n=-\infty}^{+\infty} a_{n} c\left(t-n T_{b}\right) \\
H U(t) & =\sum_{n=-\infty}^{+\infty} a_{n} e\left(t-n T_{b}\right) \\
H W(t) & =p_{3}(t) * \sum_{i=1}^{I} h_{i, t 0, \tau}^{*}(-t) * w_{i}(t) \\
U W(t) & =p_{3}(t) * \sum_{i=1}^{I} u_{i, t 0, \tau}^{*}(-t) * w_{i}(t),
\end{aligned}
$$




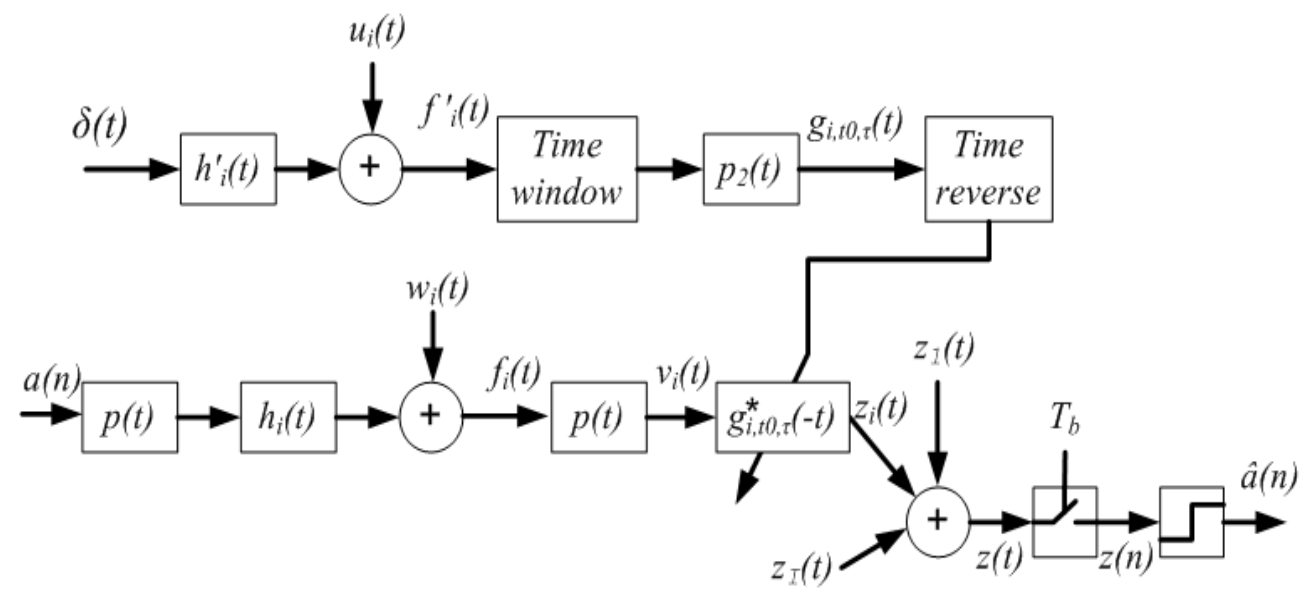

Fig. 1. Block-diagram for the application of passive time reversal to digital communications.

where

$$
\begin{aligned}
& c(t)=p_{4}(t) * \sum_{i=1}^{I} h_{i}(t) * h_{i, t 0, \tau}^{*}(-t) \\
& e(t)=p_{4}(t) * \sum_{i=1}^{I} h_{i}(t) * u_{i, t 0, \tau}^{*}(-t) .
\end{aligned}
$$

In (7) and (8) the notation selected for the noise terms revels its origin in the correlation between: $h_{i}(t)$ and $u_{i}(t)$ for $H U(t)$, $h_{i}(t)$ and $w_{i}(t)$ for $H W(t)$ and $u_{i}(t)$ and $w_{i}(t)$ for $U W(t)$.

The next logic step will be to derive the pTR output SNR using (7) and proceed to its maximization relative to the time-window parameters $t_{0}$ and $\tau$, respectively start time and duration. Before doing so, and in order to motivate this optimization procedure, the reader should consider Figures 2 and 3 that anticipate the results obtained, respectively in simulation (Section IV) and with real data (Section V). Figures 2 and 3 shows the depth dependent IRs for a reduced time scale where the sign 'o' indicates the time-window starting instant $t_{0}$, sign ${ }^{*}$ ' indicates the optimum time-window duration $\left(t_{0}+\tau_{\text {opt }}\right)$, the one that guarantees the pTR best performance as derived from the optimization of the output SNR, and sign ' + ' indicate the maximum time-window duration considered in the analysis, $t_{0}+\tau_{\max }$. Close inspection in Figure 3, for the real data noise contaminated IR estimates, reveals that as the time-window increases, more IR paths are included in $h_{i, t_{0}, \tau}(t)$ and simultaneously more noise power is included in $u_{i, t_{0}, \tau}(t)$. Those two factors will affect the pTR performance in opposite directions, resulting in an optimum time-window that does not include all the arriving paths. It should be noted however that, in order for the system to operate as a pTR, the time windowing operation must contain at least the main arrivals of the channel IRs. When operating with a vertical line array this can be done using the same time-window for all hydrophones since at long ranges, greater than a few water depths, the main arrivals approximate plane waves. Under those conditions $t_{0}$ must be set before the main arrivals and $\tau$ must include the first arriving paths. In order to proceed to the output SNR maximization one needs to first derive the various noise cross terms that will appear in the SNR expression denominator.

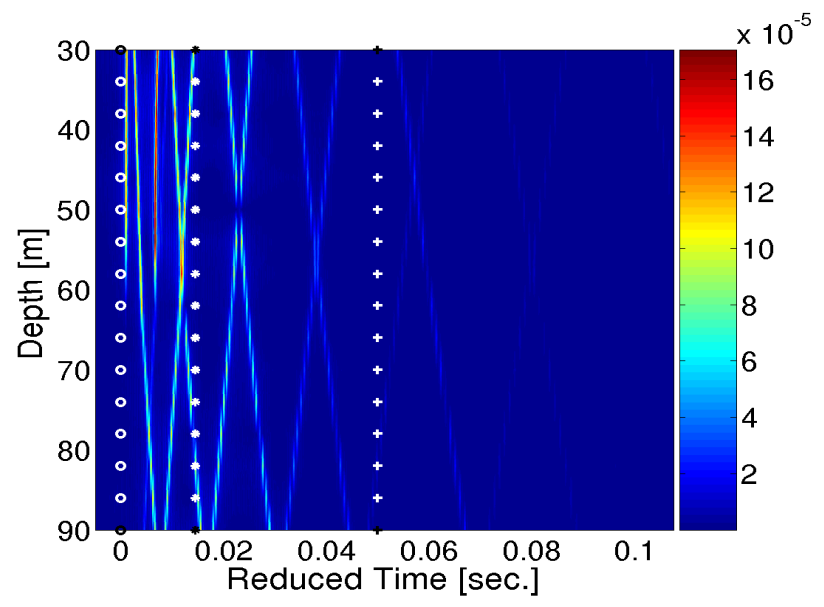

Fig. 2. Simulated depth dependent broadband arriving pattern over a realistic scenario: start time 'o', optimum window duration '*' and maximum window duration ' + '.

\section{B. Signal and noise power}

To obtain a closed form expression for the pTR SNR output it is important to characterize each noise disturbance given by (8). It can be shown that $H U(t), H W(t)$ and $L W(t)$ are zeromean Wide Sense Stationary (WSS).

The noise term $U W(t)$ results from the correlation of the two input noise terms $w_{i}(t)$ and $u_{i, t 0, \tau}(t)(8)$, where the latter is time limited by unit-gate function. It can be shown that the $U W(t)$ power is given by

$$
\sigma_{U W}^{2}(\tau)=r_{p_{3}}(0) \sigma_{w}^{2} \sigma_{u}^{2} \tau I,
$$

where $\sigma_{w}^{2}$ and $\sigma_{u}^{2}$ are the noise variances of $w(t)$ and $u(t)$ respectively, $\tau$ is the window length, $I$ is the number of hydrophones and $r_{p_{3}}\left(t^{\prime}\right)$ is the autocorrelation of $p_{3}(t)$. 


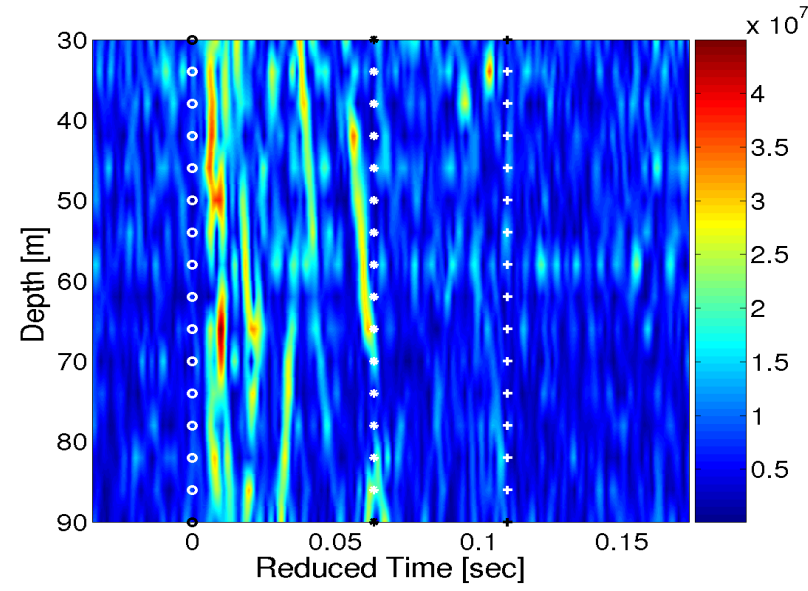

Fig. 3. Real data vertical array estimated impulse responses: start time ' 0 ', optimum window duration ' ' ' and maximum window duration ' + '.

The noise term $H W(t)$ results from the correlation of finite impulse response $h_{i, t 0, \tau}(t)$ with a stochastic signal $w_{i}(t)(8)$ and its power is given by

$$
\sigma_{H W}^{2}\left(t_{0}, \tau\right)=r_{p_{3}}(0) \sigma_{w}^{2} C_{H W}\left(t_{0}, \tau\right) .
$$

where

$$
C_{H N}\left(t_{0}, \tau\right)=\sum_{i=1}^{I} \int_{t_{0}}^{t_{0}+\tau} h_{i}(t) h_{i}^{*}(t) d t .
$$

The noise term $H U(t)$ results from the correlation of the transmitted sequence $a_{n}$, the channel IRs $h_{i}(t)$ and the noise term $u_{i, t 0, \tau}(t)$. It can be shown that its power is given by

$$
\sigma_{H}^{2}(\tau)=r_{p_{4}}(0) \frac{\sigma_{a}^{2}}{T_{b}} \sigma_{u}^{2} C_{h} \tau
$$

where

$$
C_{h}=\sum_{i=1}^{I} \int h_{i}(t) h_{i}^{*}(t) d t .
$$

is the pTR overall impulse response when the channel IRs are unlimited.

In (7) the PAM data-signal has pulse shape $c(t)$ given by (9), and its power is

$$
\sigma_{y}^{2}\left(t_{0}, \tau\right)=\frac{\sigma_{a}^{2}}{T_{b}}\left[C_{y}\left(t_{0}, \tau\right)\right]^{2} r_{p_{4}}(0)
$$

where $C_{y}\left(t_{0}, \tau\right)$ is computed in a similar manner to $C_{H W}\left(t_{0}, \tau\right)$ and becomes

$$
C_{y}\left(t_{0}, \tau\right) \delta\left(t^{\prime}\right) \approx \sum_{i=1}^{I} \int_{-\infty}^{\infty} h_{i}\left(t+t^{\prime}\right) h_{i, t_{0}, \tau}^{*}(t) d t .
$$

Under those conditions $\left[C_{y}\left(t_{0}, \tau\right)\right]^{2}$ is the autocorrelation at the origin of $C_{y}\left(t_{0}, \tau\right) \delta\left(t^{\prime}\right)$.

In the above equations the time-window dependent factors $C$.(.) that affects the signal and noise power terms are equivalent to TRM gains at the focal point for different configurations of the channel IRs (limited and/or unlimited). Those factors are related with each other and when TRM associated assumptions are fulfilled $C_{H W}$ is equal to $C_{y}$ and as $\tau$ increases they both converge to $C_{h}$.

\section{THE PASSIVE TIME-REVERSAL OPTIMIZATION}

The objective of a pTR-based demodulator is to reduce the Mean Squared Error (MSE) metric between the transmitted data symbols and the demodulated signal at the slicer input (respectively $a_{n}$ and $z_{n}$ in Figure 1). Such global metric comprises two particular metrics: the pTR output SNR metric when there is no ISI present and the pTR output ISI metric when there is no output noise present. Both, the ISI and SNR metrics, can be optimized by optimizing the time-window that was used to capture the channel IRs estimate.

\section{A. The passive time-reversal output SNR metrics}

The pTR communication system firstly recombines energy as a matched filter, whose function is to maximize the SNR in each hydrophone and then sums all $z_{i}$ signals (see Figure 1) to further reduce SNR and to reduce the ISI [9]. Considering that the array structure is adapted to the propagation environment such that the residual ISI is considered negligible the timewindowing optimization can be obtained from a closed form expression for the pTR output SNR.

The pTR communication system signal and noise power terms have already been found in (10), (11), (13) and (15). Since $H U, H W$ and $U W$ are zero mean independent random terms the variance of the sum is simply the sum of the variances and the pTR output SNR will be given by

$$
S N R_{o, \text { ideal }}\left(t_{0}, \tau\right)=\frac{\sigma_{y}^{2}\left(t_{0}, \tau\right)}{\sigma_{U W}^{2}(\tau)+\sigma_{H W}^{2}\left(t_{0}, \tau\right)+\sigma_{H}^{2}(\tau)},
$$

where its dependence on the window length, $\tau$, and starting time $t_{0}$ is perfectly clear. Such pTR output SNR is ideal in the sense that it considers that there is no residual ISI.

After the pTR, the data frame detection can be made, as in Figure 1, in two steps: by sampling the pTR output signal $z(t)$ at the symbol period, $T_{b}$, that will result in the sampled signal $z\left(n T_{b}\right)$ corrupted by noise and ISI, followed by a slicer/detector that estimates the transmitted symbols. The full elimination of the ISI can only be attained if the pTR associated assumptions are fulfilled. In a real scenario the array does not densely cover the entire water column and the overall pTR IR becomes a dirac-pulse corrupted with residual multipath that in the pTR communication system results in residual ISI.

For digital communications purpose the residual ISI should be considered as a corruption term similar to a noise term and can be incorporated in the output SNR of (17) in a similar manner of equation (33) of [9]. Despite the influence of the residual multipath over the noise terms the pTR output SNR in presence of ISI can be approximated by

$$
S N R_{o, i s i}\left(t_{0}, \tau\right) \approx \frac{\sigma_{y}^{2}\left(t_{0}, \tau\right)}{\sigma_{y}^{2}\left(t_{0}, \tau\right)\left[I S I\left(t_{0}, \tau\right)\right]+\sigma_{\text {noise }}^{2}\left(t_{0}, \tau\right)},
$$


where $\sigma_{\text {noise }}^{2}\left(t_{0}, \tau\right)=\sigma_{\text {UW }}^{2}(\tau)+\sigma_{H W}^{2}\left(t_{0}, \tau\right)+\sigma_{H}^{2}(\tau)$ and the ISI is given by the ratio between the power of the multipath spread of the pTR overall IR at the symbol rate and its main path power,

$$
I S I\left(t_{0}, \tau\right)=\frac{\sum_{n \neq 0}\left|p_{T R}\left(n T_{b}, t_{0}, \tau\right)^{2}\right|}{\left|p_{T R}\left(0, t_{0}, \tau\right)^{2}\right|}
$$

where

$$
p_{T R}\left(n T_{b}, t_{0}, \tau\right)=\left.\sum_{i=1}^{I}\left[h_{i}^{*}\left(-t, t_{0}, \tau\right) * h_{i}(t)\right] * p_{4}(t)\right|_{\substack{t=n T_{b} \\(20}}
$$

is the baseband version of the pTR IR affected by the time window operation and sampled at the symbol rate $1 / T_{b}$.

The pTR output SNR in presence of ISI given by (18) can also be computed considering the MSE between the transmitted symbols and the detector input $\overline{|a(n)-z(n)|^{2}}$ (see Figure 1) as in [9], [14]

$$
S N R_{o, m s e}\left(t_{0}, \tau\right)=\frac{1}{M S E\left(t_{0}, \tau\right)}-1
$$

When the noise power dominates over the ISI $S N R_{o, m s e}\left(t_{0}, \tau\right) \approx S N R_{o, \text { ideal }}\left(t_{0}, \tau\right)$, but when ISI dominates $S N R_{o, m s e}\left(t_{0}, \tau\right) \approx I S I^{-1}\left(t_{0}, \tau\right)$. In spite of the differences between the $S N R_{o, \text { ideal }}$ given in (17) and $S N R_{o, m s e}$ given in (21), when in presence of a well designed array that ensures $I S I^{-1}>S N R_{o, \text { ideal }}$ at the pTR output, their maxima occur for the same time-window duration, which will be clarified in Section IV. Window parameters for optimal detection can therefore be predicted from the pTR output SNR given in (17).

\section{B. Probe-timing optimization}

From (21) the global MSE metric can not be probe-timing optimized due to its depends on the transmitted data sequence, $a_{n}$, or at least can not be optimized prior to the data sequence estimate. On the other hand the ISI metric (19) can be probetiming optimized considering that the pTR overall IR estimate is available that is always the case in a pTR demodulation system. The ideal pTR SNR metric (17) depends on the channel IRs estimate and on the environmental acoustic noise level. Nevertheless, in the following it will be shown that its probe-timing optimization can be attained only considering the channel IRs estimate.

The ideal pTR SNR metric given by (17) can be simplified since in (11) and (13) $C_{H W}\left(t_{0}, \tau\right) \ll \tau C_{h}, \sigma_{w}^{2}=\sigma_{u}^{2}, \sigma_{a}^{2} / T_{b} \gg$ 1 , and $r_{p_{4}}(0)>r_{p_{3}}(0)$, such that $\sigma_{H W}^{2}\left(t_{0}, \tau\right) \ll \sigma_{H}^{2}\left(t_{0}, \tau\right)$. Then (17) reduces to

$$
\operatorname{SNR}_{o, \text { ideal }}\left(t_{0}, \tau\right) \approx \frac{\sigma_{y}^{2}\left(t_{0}, \tau\right)}{\sigma_{U W}^{2}(\tau)+\sigma_{H}^{2}(\tau)} .
$$

For values of $\tau>0$ one can define

$$
\Phi\left(t_{0}, \tau\right)=\frac{C_{y}\left(t_{0}, \tau\right)}{\tau^{\frac{1}{2}}},
$$

where $C_{y}\left(t_{0}, \tau\right)$ can be computed from (16). However for an ideal array it can be shown that $C_{y}\left(t_{0}, \tau\right)=C_{H W}\left(t_{0}, \tau\right)$ and it results that

$$
C_{y}\left(t_{0}, \tau\right)=\sum_{i=1}^{I} \int_{t_{0}}^{t_{0}+\tau}\left|h_{i}(t)\right|^{2} d t
$$

is the summation of the energy cumulative functions of the channels IRs at all hydrophones. Using (23) in (22) it results that

$$
\frac{S N R_{o, \text { ideal }}\left(t_{0}, \tau\right)}{\Phi^{2}\left(t_{0}, \tau\right)}=\frac{\left(\sigma_{a}^{2} / T_{b}\right) r_{p 4}(0)}{\sigma_{w}^{2} \sigma_{u}^{2} I r_{p 3}(0)+\left(\sigma_{a}^{2} / T_{b}\right) \sigma_{u}^{2} C_{h} r_{p 4}(0)}
$$

Since the right term of the equation is constant with $\tau, S N R_{o, \text { ideal }}\left(t_{0}, \tau\right)$ and $\left|\Phi\left(t_{0}, \tau\right)\right|^{2}$ have the same shape and the optimum $\tau$ that yields the global maximum for $S N R_{o, \text { ideal }}\left(t_{0}, \tau\right)$ is given by

$$
\tau_{\text {opt }}=\arg \max \left(\Phi\left(t_{0}, \tau\right)\right)
$$

where, with no loss of generality, the time-window starting point $t_{0}$ was considered to be chosen arbitrarily before the main path arrivals of the $h_{i}(t)$ IRs. Equations (23) and (26) state the remarkable result that the time-window that ensure the pTR maximum output SNR does not depend on the input noise power, and that it only depends on the channel IRs (see (23) and (24)). Since $C_{y}\left(t_{0}, \tau\right)$ can be seen as the overall pTR IR, $\Phi^{2}\left(t_{0}, \tau\right)$ represents its power and $\tau_{\text {opt }}$ the time-window length that guarantees an higher power of the pTR operator.

In a real situation $C_{y}\left(t_{0}, \tau\right)$ is not available since only a noisy version of $h_{i}(t)$ can be estimated in the pTR processor. In those conditions it can be shown that the optimal $\tau$ for real data is given by

$$
\begin{aligned}
\hat{\Phi}\left(t_{0}, \tau\right) & =\frac{\hat{C}_{y}\left(t_{0}, \tau\right)-\sigma_{u}^{2} I \tau}{\tau^{\frac{1}{2}}} \\
\hat{\tau}_{o p t} & =\arg \max \hat{\Phi}\left(t_{0}, \tau\right)
\end{aligned}
$$

\section{Simulation RESUlts}

The simulation scenario comprises a range independent acoustic channel with $100 \mathrm{~m}$ depth, over a $1.5 \mathrm{~m}$ thick silt subbottom and a gravel like bottom. The arrival pattern computed with the Bellhop ray/beam model [15], for a source depth of $60 \mathrm{~m}$ and a source-array range of $1.5 \mathrm{~km}$, can be seen in Figure 2, where the multipath spans over $100 \mathrm{~ms}$, with a higher concentration of energy in the first arrivals. The beginning of the time-window was chosen manually just before the first arriving path, and is represented by a vertical line of 'o' in Figure 2 where the ' + ' indicates the maximum time-window length considered in the analysis, and '*' the optimum timewindow length for pTR output SNR optimization as described below.

Two cases were considered:

1) The Low Frequency (LF) case that comprises a 16hydrophone-4-meter-spaced VLA with the first hydrophone placed at $30 \mathrm{~m}$, the transmitted data signal is a 
2-PSK PAM signal with a 50\% rolloff fourth-root raisedcosine pulse shape, the carrier frequency is of $1600 \mathrm{~Hz}$, and the data rate is $300 \mathrm{bits} / \mathrm{s}$.

2) The High Frequency (HF) case that comprises a 8hydrophone-8-meter-spaced Vertical Line Array (VLA) with the first hydrophone placed at $30 \mathrm{~m}$, the transmitted data signal is a 2-PSK PAM signal with a 50\% rolloff fourth-root raised-cosine pulse shape, the carrier frequency is of $10 \mathrm{kHz}$, and the data rate is $2000 \mathrm{bits} / \mathrm{s}$.

For the LF case a low pTR residual ISI is expected due to the low symbol rate and the high number of hydrophones. In opposition an high pTR residual ISI will be expected in the HF case. For each of the two cases Monte Carlo runs under low and high input SNR will be conducted.

Figure 4 shows the pTR output SNR (in $\mathrm{dB}$ ) as a function of window length parameterized by the input $\mathrm{SNR}\left(\mathrm{SNR}_{\mathrm{in}}\right)$, for the LF case (a) and the HF case (b). In each case, pTR output SNR results are shown via Monte-Carlo simulation with the MSE-based form (21) (' $\square$ '), using the ideal pTR closed form expression (17) (' $\nabla$ '), and for the residual ISI given by the inverse of (18) ('o').

For low residual ISI in LF case Figure 4(a) shows that for a $S N R_{\text {in }} \approx-25 \mathrm{~dB}$ good agreement is obtained between the ideal pTR and MSE curves. For high $S N R_{i n} \approx-10 \mathrm{~dB}$ the residual ISI of the TR operator becomes dominant and leads to saturation of $S N R_{m s e}$. For high residual ISI, in the HF case, Figure 4(b) shows that for a low $S N R_{\text {in }} \approx-25 \mathrm{~dB}$ good agreement in shape is obtained between the ideal pTR and MSE curves a better agreement would be obtained if instead of the $S N R_{\text {out }}$ given by (17) the $S N R_{\text {out }}$ given by (18) was used, nevertheless the agreement in shape its enough for the optimum time window length prediction. For high $S N R_{\text {in }} \approx$ $-10 \mathrm{~dB}$ the residual ISI of the TR operator becomes dominant and leads to saturation of $S N R_{m s e}$.

The overall SNR gain of the pTR is given by the array number of elements that is approximately $12 \mathrm{~dB}$ (for 16hydrophone, LF case) and 9dB (for 8-hydrophone, HF case) and the time-window length SNR and ISI improvement. In what concerns the ISI as the time-window includes more paths its tendency is to reduce as it can be observed in the enhancement of the $I S I^{-1}$ curve of Figure 4(b), nevertheless in Figure 4(a) shows that the $I S I^{-1}$ curve present a local maximum at $6 \mathrm{~ms}$ and the global maximum at $30 \mathrm{~ms}$ revealing that such tendency behave nonlinearly with the time-windowed multipath structure of the channel.

As previously mentioned for the low $S N R_{\text {in }}$ case the optimum time-window is ruled by the $S N R_{o, \text { ideal }} \approx S N R_{o, \text { mse }}$ curves, their maxima reveal that the optimum time-window length would be $15 \mathrm{~ms}$, approximately 5 symbols for the LF case, and $13.5 \mathrm{~ms}$, approximately 27 symbols for the HF case. In the high $S N R_{\text {in }}$ case the optimum time-window is ruled by the ISI and the $S N R_{o, m s e}$ curve is maximum when the $I S I^{-1}$ is maximum.

Figure 5 shows the behavior of $\Phi\left(t_{0}, \tau\right)$ (23) versus timewindow length for the LF and the HF cases. It can be seen that, as predicted by the theoretical derivation, the maxima clearly (a)

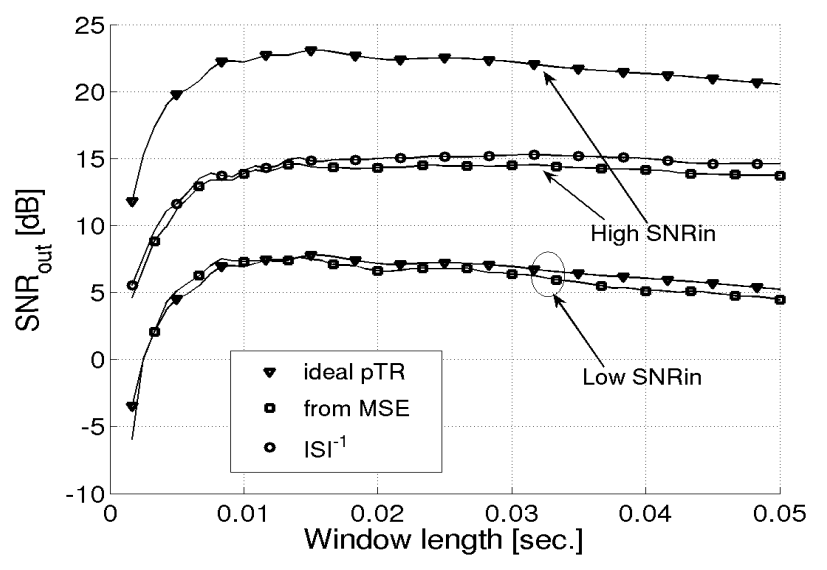

(b)

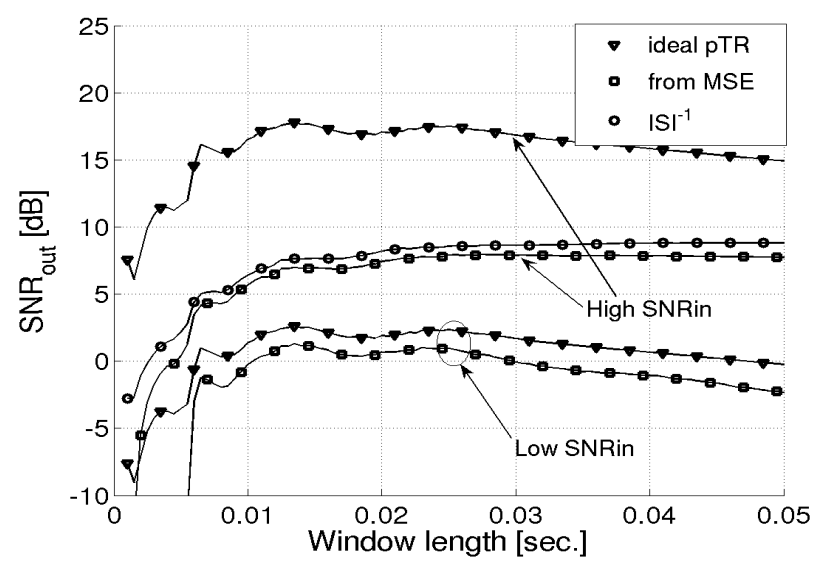

Fig. 4. Simulated pTR output SNR for the LF case (a), and for the HF case (b).

coincide with those of $S N R_{o, \text { ideal }}$ in Figure 4 both for the LF case with $\tau_{o p t}=15 \mathrm{~ms}$ and the HF case with $\tau_{o p t}=13.5 \mathrm{~ms}$. More than detecting the optimum time window when the SNR dominates over the ISI the $\Phi\left(t_{0}, \tau\right)$ curve shape agrees well with the $S N R_{o, \text { ideal }}$ and $S N R_{o, m s e}$ curves shape.

\section{EXPERIMENTAL RESUlTS}

The experimental data were acquired during the INTIFANTE'00 sea trial that took place off the town of Setúbal, approximately $50 \mathrm{~km}$ south of Lisbon (Portugal) in October 2000 [16]. This paper concentrates on the Binary Phase Shift Keying data collection. The scenario was similar to that used in Section IV with the main differences being that with real data there are noise corruption and geometric/environment mismatch between the probe-signal and the data transmissions. The acoustic source was suspended from the free drifting oceanographic vessel - NRP D. Carlos I - at a nominal depth of $60 \mathrm{~m}$. The receiver was a surface suspended 16equispaced-hydrophone vertical line array spanning nominal depths between 31 an $91 \mathrm{~m}$. The source range distance was approximately $1420 \mathrm{~m} \pm 100 \mathrm{~m}$. Nine sequential transmissions 


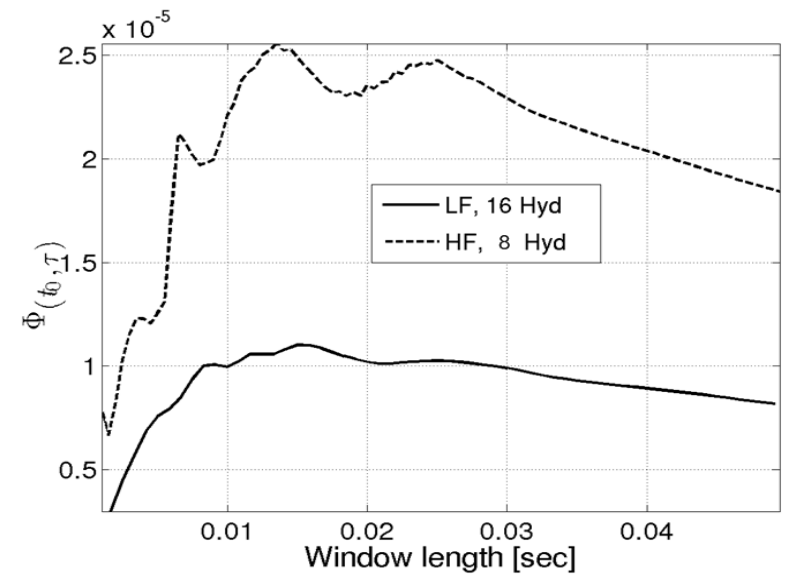

Fig. 5. Simulated performance of the proposed optimal time-window prediction method using (23) and (26) for the LF and HF cases

(in the following referred to as shot 1 to 9) will be considered, each one composed of a probe-signal transmitted 0.5 seconds before a 5 second PSK data stream, with a repetition rate of 7 seconds.

During the INTIFANTE'00 sea trial the pTR based data communications system was similar to that of Figure 1, with the $p_{2}(t)$ narrowband filter of the IR estimation operation (path above in Figure 1) distributed between the transmitter and the receiver, i.e., the transmitted probe-signal was a fourth-root raised-cosine pulse, $p_{1}(t)$, and IR estimates were obtained by pulse compression at the receiver side (see [7] for details). An example of estimated arrival pattern can be seen in Figure 3 that shows a number of arrival paths that are not as well defined as in the simulations due to noise corruption.

Figure 6(a) shows the pTR output SNR computed via the MSE at the detector input with (21), for the first 3 seconds of data during shot 7 . One can see a progressive degradation in performance due to geometric/environmental mismatch in IRs between the probe-signal and data-signal transmissions. Such loss of performance affects primarily larger time windows since those include the later arrivals that are usually considered more prone to fading. Despite this channel variability, Figure 6(b) shows that the predicted pTR output SNR maxima, given by the local maxima of $\hat{\Phi}\left(t_{0}, \tau\right)$, are in a good agrement with the true local maxima in Figure 6(a). Although the maxima location are well predicted the second and the third maxima are interchanged.

To verify the robustness of the proposed optimization technique a mean analysis over the first second of data using all nine shots is presented in Figure 7. The continuous line shows the mean pTR $S N R_{\text {out }}$ that partially eliminates the fake (noise-induced) paths and the later path arrivals that are more sensitive to fading. The dashed line shows the mean of $\hat{\Phi}\left(t_{0}, \tau\right)$ over all shots. One can see that these two curves are in excellent agreement and display an almost constant ratio, such that the same maxima locations are predicted. That suggests that pTR performance optimization is affected by (a)

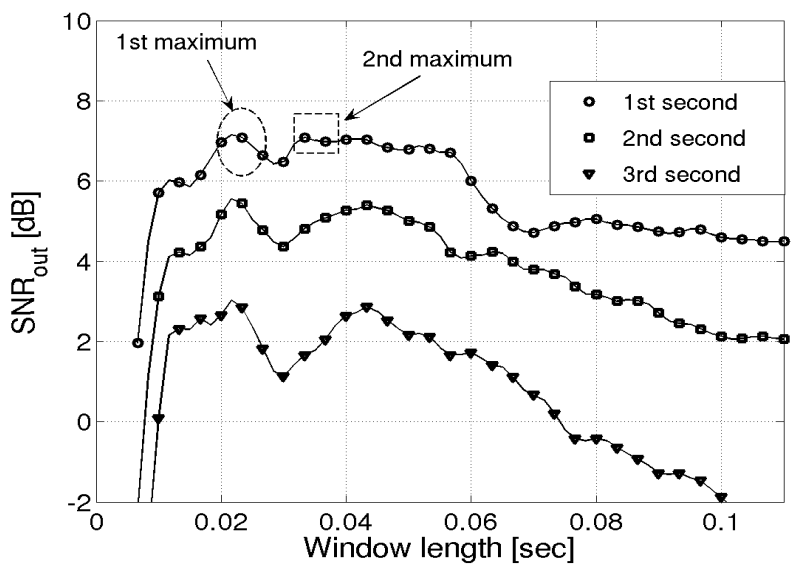

(b)

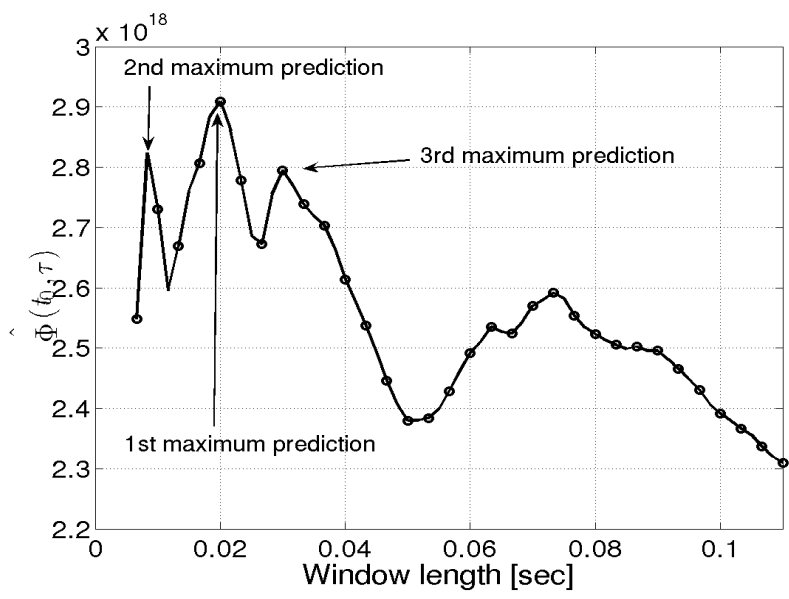

Fig. 6. Real data performance of the proposed optimal time-window prediction method obtained in shot 7: pTR output SNR computed by using the MSE at the slicer/detector input (a) and maxima prediction using (27) (b).

channel noise that will introduce a fake path structure. The problem can be overcome by enhanced IR estimation using large time-bandwidth product probe-signal or by averaging a number of closely time spaced probe-signals sent before the data stream.

Due to the good agrement between the $\hat{\Phi}\left(t_{0}, \tau\right)$ and $S N R_{o, m s e}$ curves one can state that the VLA used during the experiment is well design for the ISI reduction required to satisfy the condition that the pTR output SNR dominates over the ISI.

\section{CONCLUSION AND FUTURE WORK}

The problem of time-window optimization when operating a pTR with a VLA for underwater communications was considered. It was found that the optimum time-window simultaneously guarantees higher pTR output SNR and lower MSE at the slicer/detector input, concerning the use of a well design VLA for the environmental conditions to provide a sufficiently low ISI at the pTR output, in order to satisfy the condition that the pTR output SNR should dominate over the ISI. Time window optimization was made possible by the 


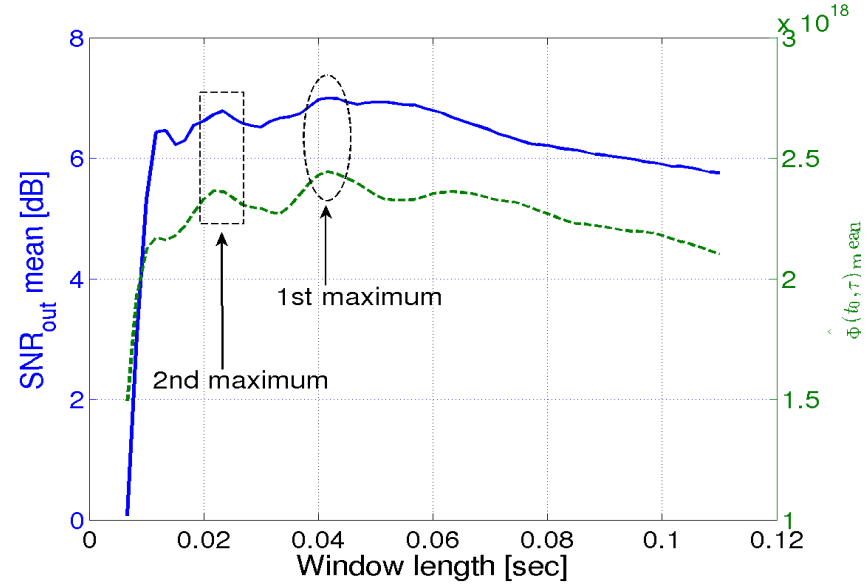

Fig. 7. Mean analysis over all shots for the real data performance of the proposed optimal time-window prediction method: pTR output SNR output computed by using the shot-mean MSE at the slicer/detector input (blue line) and shot-mean of the maxima prediction using (27) (dashed green line).

derivation of a closed-form expression for the pTR output SNR (17). Such expression allowed the derivation of (26) that clearly states that the optimum time-window depends only on the channel IRs and is not dependent on the data signal or noise level. Simulation results confirm and gauge for the theoretic foresight.

When applied to real data the channel IRs are not available and noisy estimates must be used. Even with heavily noise corrupted IRs the developed technique presents a good fit with the pTR output SNR and its global maximum being closely predicted in most of the shots. Noise-related problems in IRs estimation are mainly due (in real data) to the use of low power probe-signals (fourth-root raised cosine pulse). The usage of high power probe-signals such as chirp signals or Msequences should be addressed in future experiments. Despite its quality, it was found that the optimum time-window loses validity after a few seconds due to geometric/environment variability. Future developments should address the problem of using the proposed time-window optimization with adaptive pTR-based equalizers [17]. Up to now the proposed probe timing optimization method as been successfully applied with the pTR based environment equalizers presented in [18].

Although it was developed for pTR, the time-window optimization method can also be applied to aTR by considering that in the latter case the noise term $U W$ does not exist and $H W$ and $H U(8)$ are slightly different (see [5]).

\section{ACKNOWLEDGMENTS}

The authors would like to thank the NATO Undersea Research Centre (NURC) for the loan of the acoustic sound source, the support of Enrico Muzzi and the NRP D. Carlos I crew during the INTIFANTE'00 sea trial. This work was partially funded by FCT (ISR/IST plurianual funding) through the PIDDAC Program funds and by the FCT projects PHITOM [PTDC/EEA-TEL/71263/2006] and ATOMS [POCTI/P/MAR/15296/1999].

\section{REFERENCES}

[1] M. Stojanovic, J. Catipovic, and J. Proakis. Adaptive multichannel combining and equalization for underwater acoustic communications. J. Acoust. Soc. America, 94(3):1621-1631, 1993.

[2] R.D. Jackson and R.D. Dowling. Phase conjugation in underwater acoustics. J. Acoust. Soc. Am., 89(1):171-181, January 1991.

[3] R.D. Dowling. Acoustic pulse compression using passive phaseconjugate processing. J. Acoust. Soc. America, 95(3):1450-1458, 1994.

[4] A. Silva, S. Jesus, J. Gomes, and V. Barroso. Underwater acoustic communications using a 'virtual' electronic time-reversal mirror approach. In P. Chevret and M.Zakharia, editors, 5th European Conference on Underwater Acoustics, pages 531-536, Lyon, France, June 2000.

[5] J. Gomes and V. Barroso. Asymmetric underwater acoustic communication using a time-reversal mirror. In Proc. of the MTS/IEEE Oceans 2000, Providance, USA, 2000.

[6] D. Rouseff, L.J.W. Fox, D. Jackson, and D.C. Jones. Underwater acoustic communications using passive phase conjugation. In Proc. of the MTS/IEEE Oceans 2001, pages 2227-2230, Honolulu, Hawai, USA, November 2001.

[7] A. Silva and S. Jesus. Underwater communications using virtual time-reversal in a variable geometry channel. In Proc. MTS/IEEE Oceans'2002, pages 2416-2421, Biloxi, USA, November, 2002.

[8] T. C. Yang. Differences between passive-phase conjugation and decision-feedback equalizer for underwater acoustic communications. IEEE Journal of Oceanic Engineering, 29(2):472-487, April 2004.

[9] M. Stojanovic. Retrofocusing techniques for high rate acoustic communications. J. Acoust. Soc. America, 117(3):1173-1185, 2005.

[10] W.A. Kuperman, W. Hodgkiss, H. Chun Song, T. Akal, C. Ferla, and D. Jackson. Phase conjugation in the ocean: Experimental demonstration of an acoustic time-reversal mirror. J. Acoust. Soc. Am., 103(1):25-40, January 1998.

[11] G.F. Edelmann, W.S. Hodgkiss, S. Kim, W.A. Kuperman, H.C. Song., and T. Akal. Underwater acoustic communications using time-reversal. In Proc. of the MTS/IEEE Oceans 2001, pages 2231-2235, Honolulu, Hawai, USA, 5-8 November 2001.

[12] J.V. Candy, A.M. Meyer, A.J. Poggio, and B.L. Guidry. Time-reversal processing for an acoustic communications experiment in a highly reverberant environment. J. Acoust. Soc. America, 115(4):1621-1631, 2004.

[13] A. Silva. Environmental based underwater communications. Phd. dissertation, Instituto Superior Tecnico, Lisboa, Portugal, 2009.

[14] J.G. Proakis. Digital Communications. McGraw-Hill, Massachusetts, 1995.

[15] F. Jensen, W. Kuperman, M. Porter, and H. Schmidt. Computational Ocean Acoustics. AIP Series in Modern Acoustics and Signal Processing, New York, 1994.

[16] S.M. Jesus and A. Silva. Virtual time reversal in underwater acoustic communications: Results on the intifante'00 sea trial. In Proc. of Forum Acusticum, Sevilla, Spain, September 2002.

[17] J. Gomes, A. Silva, and S.M. Jesus. Adaptive spatial combining for passive time-reversed communications. J. Acoust. Soc. America, 124(2):1038-1053, August 2008.

[18] A. Silva, S.M. Jesus, and J. Gomes. Environment-based underwater communications. In Proc. of the OCEANS'07, Vancouver, Canada, October 2007. 